\title{
Two Exceptional Complications Revealing Celiac Disease: Ischemic Cardiomyopathy and Pellagra
}

\author{
Imed Ben Ghorbel ${ }^{1}$, Raouf Hajji ${ }^{2, *}$, Nabil Bel Feki ${ }^{1}$, Thouraya Ben Salem ${ }^{1}$, Mounir Lamloum ${ }^{1}$, \\ Mohammed Habib Houman ${ }^{1}$ \\ ${ }^{1}$ Internal Medicine Department, University Hospital la Rabta, Tunis, Tunisia \\ ${ }^{2}$ Internal Medicine Department, Sidi Bouzid Regional Hospital, Sidi Bouzid, Tunisia \\ *Corresponding author: raouf.hajji@yahoo.fr \\ Received January 11, 2015; Revised January 15, 2015; Accepted January 17, 2015
}

\begin{abstract}
Pellagra is an exceptional skin involvement in celiac disease. Ischemic cardiomyopathy is another rare event in celiac disease. Its pathogenesis remains not yet elucidated. We report a case of a 34- year old patient of presenting with pellagra and cardiomyopathy revealing a celiac disease.
\end{abstract}

Keywords: pellagra, ischemic heart disease, celiac disease

Cite This Article: Imed Ben Ghorbel, Raouf Hajji, Nabil Bel Feki, Thouraya Ben Salem, Mounir Lamloum, and Mohammed Habib Houman, "Two Exceptional Complications Revealing Celiac Disease: Ischemic Cardiomyopathy and Pellagra.” International Journal of Celiac Disease, vol. 3, no. 1 (2015): 31-32. doi: 10.12691/ijcd-3-1-5.

\section{Introduction}

Pellagra (or "rough skin,” from the Italian pelle agra) is an exceptional skin involvement in celiac disease due to tryptophan and B3 vitamin (niacin) deficiency [1]. Ischemic cardiomyopathy is another rare event in celiac disease [2]. Its pathogenesis remains not yet elucidated. We report a case of a patient presenting with pellagra and cardiomyopathy revealing a celiac disease.

\section{Case Report}

A 34-year-old male patient was admitted in intensive care unit of cardiology in 2009 for acute chest pain. He was not smoker neither alcohol consumer. He had no family nor personal history of coronary artery disease. The myocardial infarction was diagnosed and coronary angiography concluded to severe stenosis of the anterior interventricular artery. It was rapidly complicated by severe heart failure. The heart ultrasound imaging has showed dilated cardiomyopathy with systolic ejection fraction at $25 \%$. The patient was treated by aspirin, ramipril, simvastatin, and nebivolol.

Three years later, he developed diffuse red skin lesions on the face and hands (Figure 1), beefy red glossitis and high skin sensitivity to sunlight. He reported also chronic diarrhea with 4 or more watery stools/ day for several months. He presented impaired general condition with moderate asthenia and weight loss. Physical examination showed mitral holosystolic murmur and lower limbs edema. Dermatologic examination revealed desquamation, erythema, and keratosis of sun-exposed areas especially on the face, hands and feet.

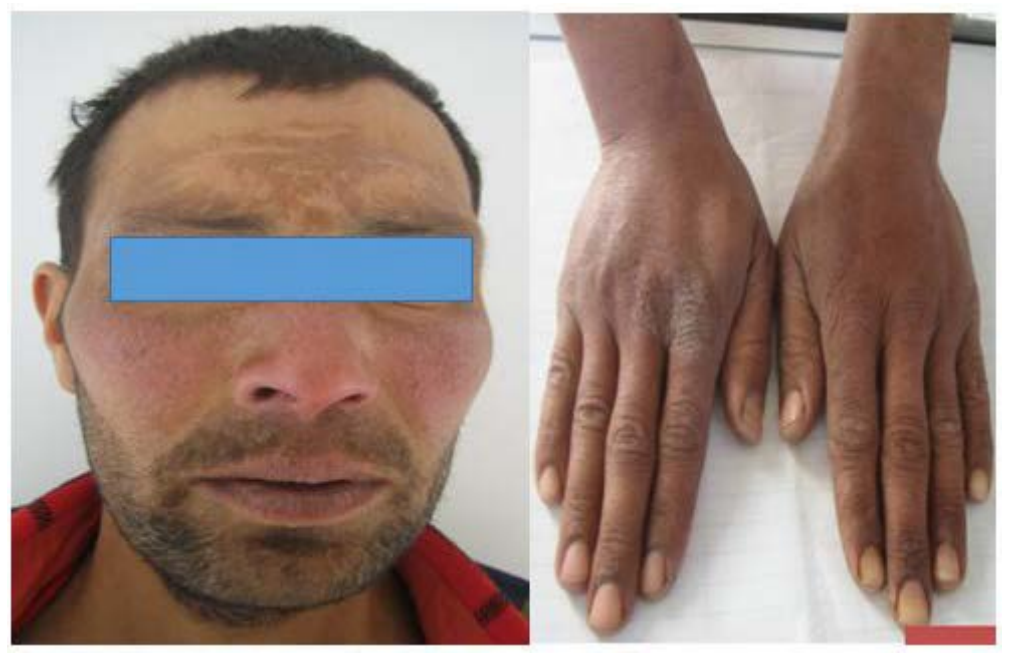

Figure 1. Lesions of pellagra: diffuse red skin lesions on the face and hands 
The complete blood count, ferritin, calcemia, the serum protein electrophorosis and lipid profile were normal. The homocysteine rate was $23 \mu \mathrm{mol} / \mathrm{l}$ (Normal: $15 \mu \mathrm{mol} / \mathrm{l}$ ).

Antiendomysial (EMA) antibodies were positive. Antitissue transglutaminase antibodies (TTGA) (Normal: 4 to $10 \mathrm{U} / \mathrm{L}$ ) were positive with a rate at $17 \mathrm{U} / \mathrm{L}$. Anti-nuclear antibodies and antiphospholipid antibodies were negative. Oesogastroduodenal fibroscopy showed flattened duodenal folds. The histology of duodenal biopsies had revealed a subtotal mucous atrophy type $3 \mathrm{~b}$ in Marsh modified (Oberhuber) histological classification of celiac disease: Increased intraepithelial lymphocytes, crypt hyperplasia and subtotal villous atrophy.

Although B3 vitamin and tryptophan dosage were not performed, the diagnosis of pellagra complicating celiac disease was retained. A gluten-free diet was introduced. The patient died of fatal cardiac failure one month after time of diagnosis: he had not followed this diet.

\section{Discussion}

Although pellagra used to be a disease of epidemic proportions in the developed world, it is now a rare condition confined to certain at-risk groups. Little progress has been made in our knowledge of pellagra since it was first described by Gaspar Casal in 1735 [3,4] and since Goldberger discovered that nicotinamide was a preventive factor in 1926 [5,6].

Proof of this is that there have been no changes in treatment or diagnostic criteria in the last 90 years.

Niacin (B 3 vitamin) forms into nicotinic acid and nicotinamide, which serve as precursors of two important coenzymes of cellular metabolism, namely nicotinamide adenine dinucleotide (NAD) and NAD-phosphate (NADPH). The deficiency of these two coenzymes is the basis of pellagra pathogenesis. In fact, NAD and NADPH participate in multiple mechanism in cell metabolism: the metabolism of amino acids and proteins (tryptophane and B3 vitamin (niacin) absoprtion), the generation of highenergy phosphate bonds and glycolysis $[7,8]$.

Thus, because of their deficiency, pellagra is essentially affecting tissues with a high rate of cell turnover, such as the digestive tract and the skin, and tissues with high energy needs, such as the brain [9,10,11].

In our case, deficiency of NAD and NAD phosphate (tryptophan and B3 vitamin) was due to malabsorption in celiac disease as it was reported by Schattner in 1999 [1].

Ischemic cardiomyopathy in celiac disease could be due to hyperhomocysteinemia. Makhdoom et al recently described a patient with idiopathic dilated cardiomyopathy, celiac disease and anticardiolipin syndrome presenting a dramatic cardiac improvement [12].
In our case, antiphospholipid antibodies were negative.

In literature, there are few cases of myocarditis that can be reversible as in the case of Amira $\mathrm{H}$ et al or complicated by dilated cardiomyopathy without vascular involvement. The outcomes of the heart and the skin involvement of celiac disease depends much free-gluten diet adherence [13].

Our patient was marked by progressive worsening till to death because he didn't follow the gluten-free diet.

\section{Conclusion}

This case illustrates two rare complications revealing celiac disease in an adult patient. Pellagra, a vitamin deficiency disease, is caused in this case by chronic malabsorption of B3 vitamin or tryptophan. Ischemic cardiomyopathy is probably due to an accelerated atherosclerosis in a persistent inflammatory disease.

\section{References}

[1] Schattner A. A 70-year-old man with isolated weight loss and a pellagra-like syndrome due to celiac disease. Yale J Biol Med. 1999; 72: 15-18.

[2] Emilsson L, Carlsson R, James S, Hambraeus K, Ludvigsson JF. Follow-up of ischaemic heart disease in patients with coeliac disease. Eur J Prev Cardiol. 2015; 22(1): 83-90.

[3] Major RH: Classic Descriptions of Disease. Springfield, Ill, Charles C. Thomas, 3rd Ed, 1945, pp 607-615.

[4] Major RH: Don Gasper Casal, Francois Thiery and pellagra. Bull Hist Med 1944; 16: 351-361.

[5] Goldberger J, Wheeler GA, Lillie RD, et al: A further study of butter, fresh beef, and yeast as pellagra preventives, with consideration of the relation of factor P-P of pellagra (and black tongue of dogs) to vitamin B. Public Health Rep 1926; 41:297-318

[6] Goldberger J: Pellagra. Essays on History of Nutrition and Dietetics. Chicago, American Dietetic Association, 1967, pp 103106.

[7] Piqué-Duran E, Pérez-Cejudo JA, Cameselle D, Palacios-Llopis S, García-Vázquez O. Pellagra: a clinical, histopathological, and epidemiological study of 7 cases. Actas Dermosifiliogr. 2012; 103(1): 51-8.

[8] Wan P1, Moat S, Anstey A. Pellagra: a review with emphasis on photosensitivity. Br J Dermatol. 2011; 164(6): 1188-200.

[9] Hegyi J, Schwartz RA, Hegyi V. Pellagra: dermatitis, dementia and diarrhea. Int J Dermatol. 2004; 43:1-5.

[10] Pitche PT. Pellagre et érythèmes pellagroïdes. Cahiers Sánte. 2005; 15: 205-8.

[11] Nogueira A, Duarte AF, Magina S, Azevedo F. Pellagra associated with esophageal carcinoma and alcoholism. Dermatol Online J. 2009; 15: 8.

[12] Makhdoom ZA, Randall NW. Dilated cardiomyopathy due to anticardiolipin syndrome in association with celiac sprue. J Clin Gastroenterol. 2000; 31(1): 91-2.

[13] Amira H, Raouf H, Syrine B, Haifa N, Monia SK, Slim H, Habib HM. Atypical presentation of adult celiac disease: myocarditis and liver abnormality. Anadolu Kardiyol Derg. 2013; 13(6): 615-6. 